\title{
GENETIC ALGORITHM WITH SRM SVM Classifier For FACE VERIFICATION
}

\author{
Safiya K.M ${ }^{1,}$ Prof.S.Bhuvana ${ }^{2,}$ Prof.P.TamijeSelvy ${ }^{3}$ and Dr. R. Radhakrishnan ${ }^{4}$ \\ ${ }^{1}$ PG Student, Department of Computer Science and Engineering, Sri Krishna College of \\ Technology, Coimbatore, India, \\ safiyamoideen@gmail.com \\ ${ }^{2}$ Assistant Professor, Department of Computer Science and Engineering, Sri Krishna \\ College of Technology, Coimbatore, India, \\ bhuvana_anju@rediffmail.com \\ ${ }^{3}$ Assistant Professor, Department of Computer Science and Engineering, Sri Krishna \\ College of Technology, Coimbatore, India, \\ tamijeselvy@gmail. com \\ ${ }^{4}$ Principal, Sri Ramakrishna Engineering college, Coimbatore. \\ rlgs14466@gmail.com
}

\begin{abstract}
Face verification is an important problem. The problem of designing and evaluating discriminative approaches without explicit age modelling is used. To find the gradient orientation discard magnitude information. Using hierarchical information this representation can be further improved which results in the use of gradient orientation pyramid. When combined with a structural risk minimization support vector machine with genetic algorithm, gradient orientation pyramid demonstrate excellent performance. Gradient Orientation of each color channel of human faces is robust under age progression. The feature vector which is computed as the cosines of the difference between gradient orientations at all pixels, is given as the input to the structural risk minimization support vector machine classifier. The classifier is used to divide the feature space into two classes, one for the intrapersonal pairs and the other for extrapersonal pairs. Genetic algorithm plays an important role in improving the performance of the system. The system outperformed other classifiers such as support vector machine and boosting support vector machine.
\end{abstract}

\section{KEYWORDS}

Face verification, feature space, genetic algorithm, gradient orientation pyramid(GOP), structural risk minimization, support vector machine(SVM).

\section{INTRODUCTION}

Face verification is an important problem in computer vision and has very wide range of applications such as surveillance, human-computer interaction, image retrieval. Discriminative approaches have been used for face verification across age progression. Gradient orientation pyramid representation framework is suitable for face verification[1]. Structural Risk minimization support vector machine(SRM-SVM)[8] with genetic algorithm (GA) is proposed for the classification. The classifier classifies the image pair as intrapersonal(images are from the same individual) or extra personal(images are from different individual). The facial images are taken from the FG-NET database. 


\subsection{Gradient Orientation Pyramid}

A hierarchical representation is made by combining the gradient directions at different scales. The Gradient Orientation Pyramid has several advantages. Gradient Orientation Pyramid is insensitive to illumination changes[1]. No normalization is needed on the input images. GO is robust across age progression for face verification tasks where high resolution images are avoided. The Technique provides a natural way to perform face comparison at different scales[1].

Find the gradient orientation at all pixels of the image[2] An image pair and corresponding gradient orientation pyramids are taken, the feature vector is computed as the cosines of the difference between gradient orientations at all pixels over scale. Apply Gaussian kernel to the extracted feature. The feature vector is computed using both the images. Consider gradient orientation at all pixels of the image.

\subsection{Classification}

Classification includes a broad range of decision-theoretic approaches to the identification of images . All classification algorithms are based on the assumption that the image in question depicts one or more features and that each of these features belongs to one of several distinct and exclusive classes.

The classes specified a priori by an analyst or automatically clustered into sets of prototype classes, where the analyst merely specifies the number of desired categories.

Image classification analyzes the numerical properties of various image features and organizes data into categories. Classification algorithms typically employ two phases of processing: training and testing. In the initial training phase, characteristic properties of typical image features are isolated and, based on these, training class, is created. In the subsequent testing phase, these feature-space partitions are used to classify image features.

The description of training classes is an extremely important component of the classification process. In supervised classification, statistical processes or distribution-free processes can be used to extract class descriptors. Unsupervised classification relies on clustering algorithms to automatically segment the training data into prototype classes.

\section{RELATED WORK}

\subsection{ILLUMINATION INVARIANTS}

Consider the problem of determining functions of an image of an object that are insensitive to illumination changes. First show that for an object with Lambertian reflectan no discriminative functions that are invariant to illumination. This result leads us to adopt a probabilistic approach in which we analytically determine a probability distribution for the image gradient as a function of the surfaces geometry and reflectance. Our distribution reveals that the direction of the image gradient is insensitive to changes in illumination direction.

Verify empirically by constructing a distribution for the image gradient from more than twenty million samples of gradients in a database of thousand two hundred and eighty images of twenty inanimate objects taken under varying lighting conditions. Using this distribution, develop an illumination insensitive measure of image comparison and test it on the problem of face recognition. 


\subsection{Support Vector Machine}

Face recognition is a $\mathrm{K}$ class problem, where $\mathrm{K}$ is the number of known individuals; and support vector machines (SVMs) are a binary classification method. By reformulating the face recognition problem and reinterpreting the output of the SVM classifier, we developed a SVM-based face recognition algorithm.

The face recognition problem is formulated as a problem in difference space, which models dissimilarities between two facial images. In difference space we formulate face recognition as a two class problem. The classes are: dissimilarities between faces of the same person, and dissimilarities between faces of different people.

By modifying the interpretation of the decision surface generated by SVM, we generated a similarity metric between faces that is learned from examples of differences between faces. The SVM-based algorithm is compared with a principal component analysis (PCA) based algorithm on a difficult set of images from the FERET database.

Performance was measured for both verification and identification scenarios. The identification performance for SVM is $77-78 \%$ versus $54 \%$ for PCA. For verification, the equal error rate is $7 \%$ for SVM and $13 \%$ for PCA.

Support Vector Machines (SVMs) are formulated to solve a classical two class pattern recognition problem. The system adapt SVM to face recognition by modifying the interpretation of the output of a SVM classifier and devising a representation of facial images.

\subsection{Face Recognition As People Age}

Study face recognition across ages within a real passport photo verification task. Propose using the gradient orientation pyramid for this task. Discarding the gradient magnitude and utilizing hierarchical techniques, found that the new descriptor yields a robust and discriminative representation. With the proposed descriptor, model face verification as a two-class problem and use a support vector machine as a classifier.

The approach is applied to two passport data sets containing more than 1,800 image pairs from each person with large age differences. Although simple, our approach outperforms previously tested Bayesian technique and other descriptors, including the intensity difference and gradient with magnitude.

In addition, it works as well as two commercial systems. Second, for the first time, empirically study how age differences affect recognition performance. Experiments show that, although the aging process adds difficulty to the recognition task, it does not surpass illumination or expression as a confounding factor.

\subsection{Face Verification Across Age Progression}

Human faces undergo considerable amounts of variations with aging. While face recognition systems have been proven to be sensitive to factors such as illumination and pose, their sensitivity to facial aging effects is yet to be studied.

How does age progression affect the similarity between a pair of face images of an individual. What is the confidence associated with establishing the identity between a pair of age separated 
face images. Develop a Bayesian age difference classifier that classifies face images of individuals based on age differences and performs face verification across age progression.

Study the similarity of faces across age progression. Since age separated face images ages invariably differ in illumination and pose, propose preprocessing methods for minimizing such variations.

Experimental results using a database comprising of pairs of face images that were retrieved from the passports of 465 individuals are presented. The verification system for faces separated by as many as nine years, attains an equal error rate of $8.5 \%$.

\section{PROPOSED SYSTEM}

The proposed system use gradient orientation pyramid and SRM-SVM with genetic algorithm. Differs from existing system in both the representation and the classification framework. Determine whether two images come from the same person. Does not require many images for each subject.

Face verification in the presence of age progression is an important problem. The system addresses the problem by designing and evaluating discriminative approaches without explicit age modeling. First this approach find the gradient orientation after discarding magnitude information. This representation is further improved when hierarchical information is used which results in the use of gradient orientation pyramid. When combined with a support vector machine gradient orientation pyramid demonstrate excellent performance.

To use a genetic algorithm, represent a solution to problem as a genome (or chromosome). The genetic algorithm then creates a population of solutions and applies genetic operators such as mutation and crossover to evolve the solutions in order to find the best one(s). This presentation outlines some of the basics of genetic algorithms. The three most important aspects of using genetic algorithms are: (1) definition of the objective function, (2) definition and implementation of the genetic representation, and (3) definition and implementation of the genetic operators. Once these three have been defined, the generic genetic algorithm should work fairly well. Beyond that you can try many different variations to improve performance, find multiple optima (species - if they exist), or parallelize the algorithms.

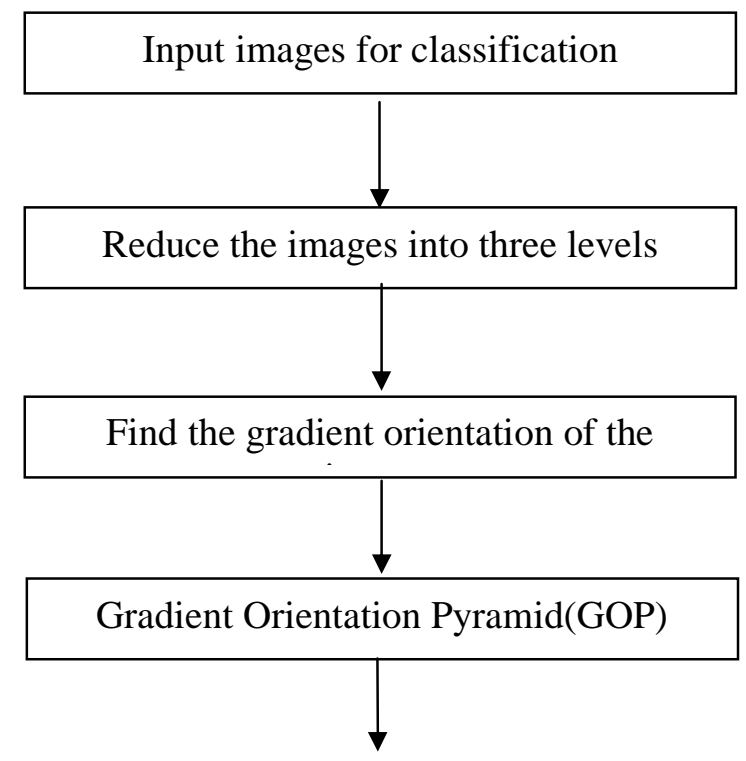




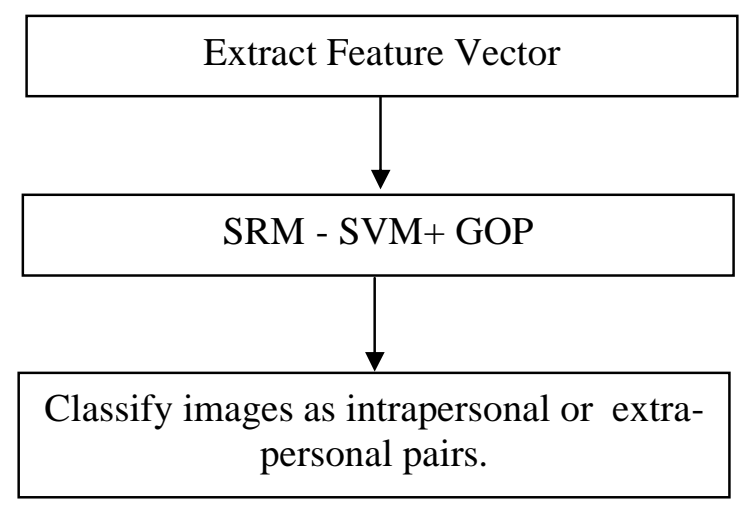

Fig1: Overall system design

\subsection{GRADIENT ORIENTATION PYRAMID}

To get better result first construct a pyramid for the images. Gaussian kernel is used. Reduce the image into three levels. Find the gradient orientation at each scale is find out using 'gradient' function. Plot the original image and three reduced images on the screen.[1]

\subsection{Kernels Between GOPs}

Find the gradient orientation at all pixels of the image. Take an image pair and corresponding gradient orientation pyramids, the feature vector is computed as the cosines of the difference between gradient orientations at all pixels over scale. Here use Gaussian kernel to the extracted feature. The feature vector is computed using both the images. Consider gradient orientation at all pixels of the image.[1]

\subsection{SVM CLASSIFICATION}

A support vector machine constructs a hyperplane or set of hyperplanes in a high- or infinitedimensional space, which can be used for classification. A separation is achieved by the hyperplane that has the largest distance to the nearest training data points of any class (so-called functional margin), since in general the larger the margin the lower the generalization error of the classifier .[5]. SVM is a binary classifier and it is immune to noise.

\subsection{BOOSTING SVM CLASSIFICATION}

Boosting SVM cascade is trained using a boosting process. In this the number of features in a given stage, increases with respect to the preceding stage. The complexity of the cascade and its classification capability increase with respect to the number of stages of the structure. A simple training method is employed in Boosting-SVM where each stage of the cascade is trained in such way that a constant true positive rate is achieved by adjusting the values of svm in the current stage. The number of features is incremented as the number of stages increases. The number of features is selected to be constant.[8] 


\subsection{SRM-SVM WITH GENETIC ALGORITHM}

An inductive principle is used in Structural risk minimization (SRM). It is used for model selection used for learning from finite training data sets. It describes a general model of capacity control and provides a trade-off between hypothesis space complexity and the quality of fitting the training data . [8]

The inductive principle called structural risk minimization (SRM) is a formal method for choosing an optimal model complexity for a finite sample. SRM has been originally proposed and applied for classification, it is applicable to any learning problem where the risk functional has to be minimized. A priori specification of a structure on a set of approximating function is required in SRM for solving a learning problem with finite data.

SRM-SVM (Structural Risk Minimization Support Vector Machine) is used as the classifier. The SRM cascade classifier follows a learning strategy which selects a suitable number of features for each ensemble stage. The selection is in such a way as to control the complexity to minimize the structural risk of each classification stage.

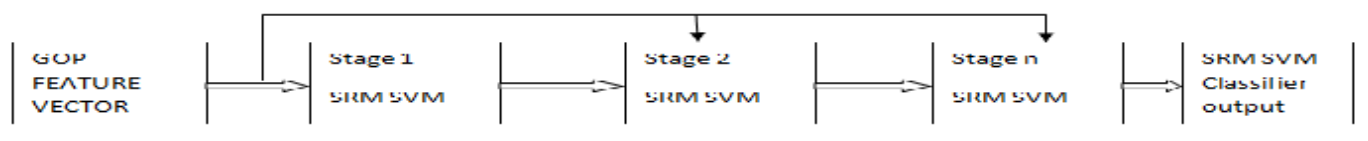

Fig 2: SRM-SVM Classifier

Genetic Algorithm methods for optimization are widely used in computer field[11].

A learning strategy is followed in SRM-SVM which selects a number of features for each stage. $\mathrm{VCd}$ is used as the complexity controller of the classifier. First construct a representation framework by reducing and finding the gradient of the images.Then the feature vector extracted from the images which act as an input to the SRM SVM classifier. The GA is added with SRM SVM to get best result. The GA consists of following steps

1. Initialization

2. Selection

3. Crossover

4. Mutation

5. Stop condition.

GA Algorithm is explained below.

- First set the number of iterations

- Then Initialize population for iteration

- Evaluate fitness function

- Start the loop and perform crossover and mutation operation.

- Stop when termination condition meets. 
International Journal of Computer Science \& Information Technology (IJCSIT) Vol 4, No 4, August 2012

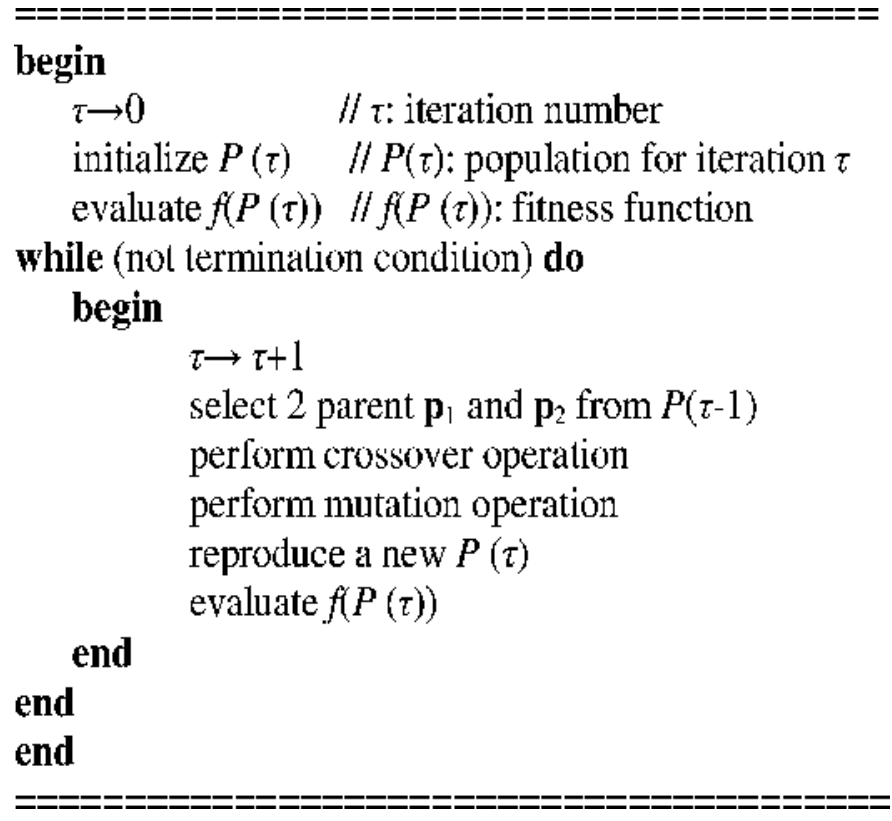

Algorithm 1: Basic steps in GA

The crossover operation exchanges information from the two parents, chromosomes $\mathbf{p}_{1}$ and $\mathbf{p}_{2}$, obtained in the selection process with a defined probability of crossover ${ }_{c}$. This probability gives an expected number of chromosomes that take part in the crossover.

\section{EXPERIMENTATION}

The experiment was carried out using the FG-NET Aging Database. It is an image database containing face images showing a number of subjects at different ages. There are 1002 images belonging to 82 subjects with an average of 12 images per subject. The images have different illumination, pose, expression, and includes faces with beards, moustaches, spectacles, and hats. 


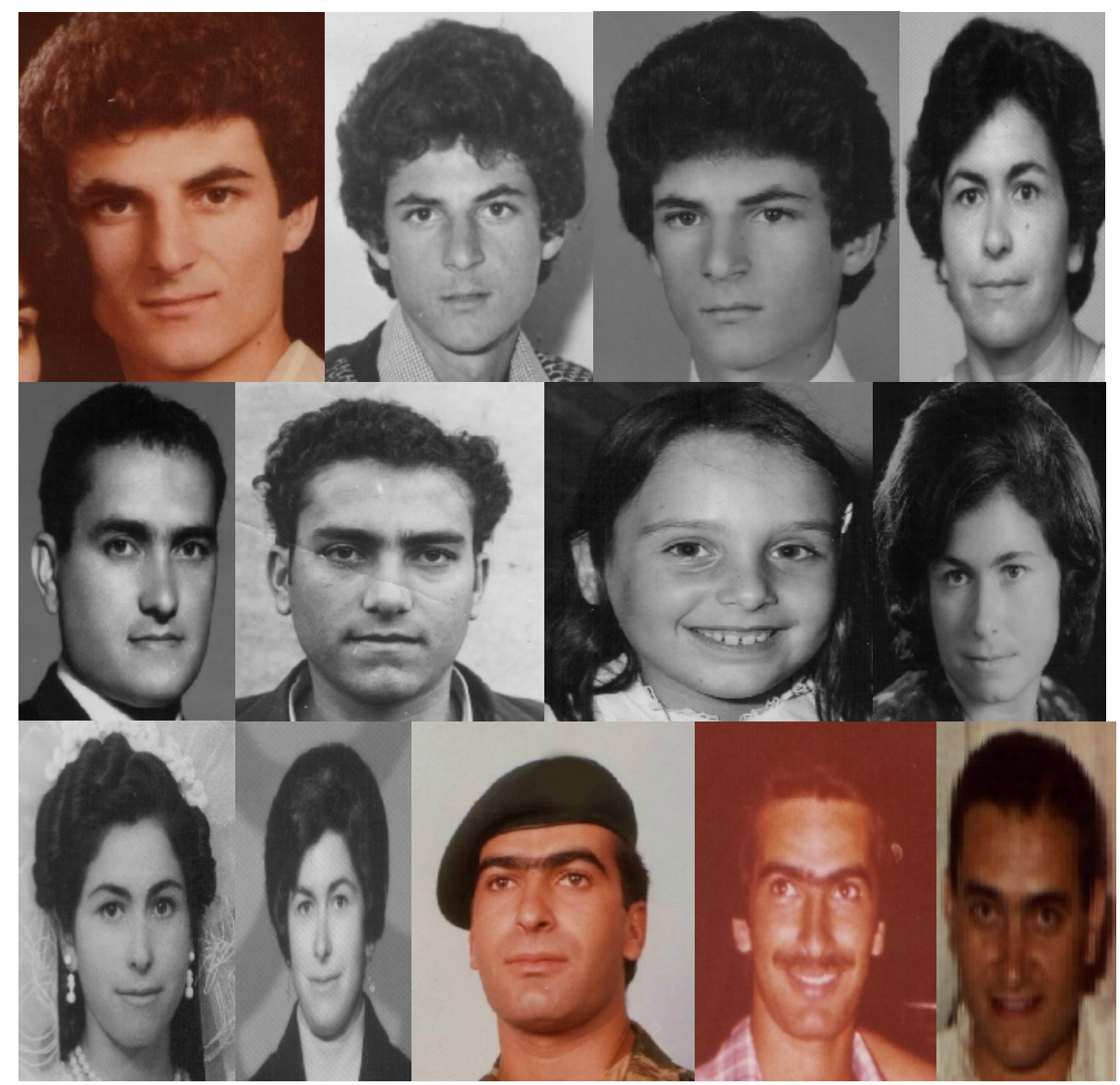

Fig 3 Some of the images from FG-Net Database.

The input is given as images which are taken at different times. First the images in the database are trained. The input is compared with those images which are in the database. The classifier will classify the images into whether they are belongs to the same person or belongs to different person.

The input is converted into its pyramid representation by converting it into its first level, second level and third level pyramid representation. Then find out the gradient representation of each reduced level images. The input image and the three level reduced images are shown in the below figure. For the input pair of images feature vector is computed as the cosines of the difference between gradient orientations at all pixels of the image. Then it is applied to SRM-support vector machine classifier. SRM-SVM do the training first and then classify the image pair. 
International Journal of Computer Science \& Information Technology (IJCSIT) Vol 4, No 4, August 2012

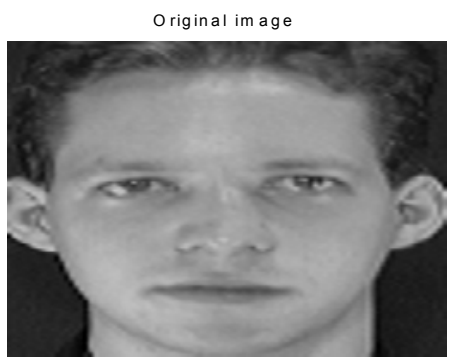

$1 \mathrm{st}$ P y ram id im age

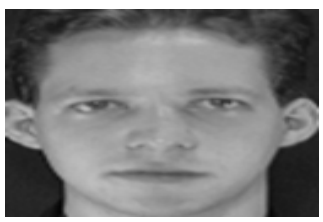

$2 \mathrm{nd} P$ yram id im age

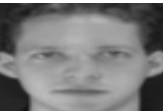

3 rd $P$ y ram id im a ge

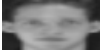

\section{Gradient Orientation

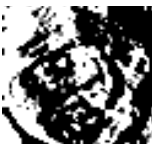

Fig4: Sample Input image, reduced level images and gradient orientations. 
International Journal of Computer Science \& Information Technology (IJCSIT) Vol 4, No 4, August 2012

The extracted feature vector values are shown in figure4.

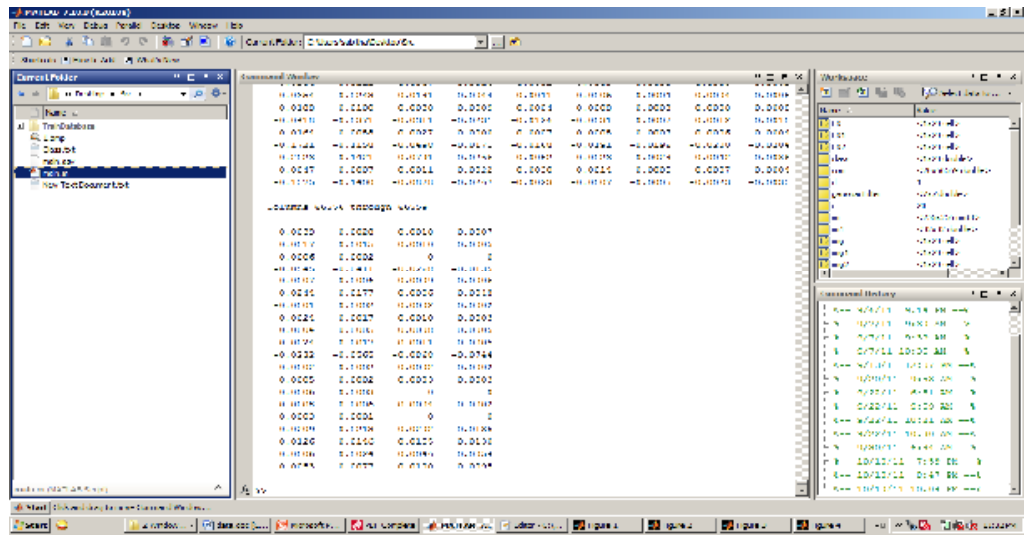

Fig5: Kernels between gops

The support vector machine classifies the images into extra personal pairs and intrapersonal pairs. The output of the classifier is shown figure5.

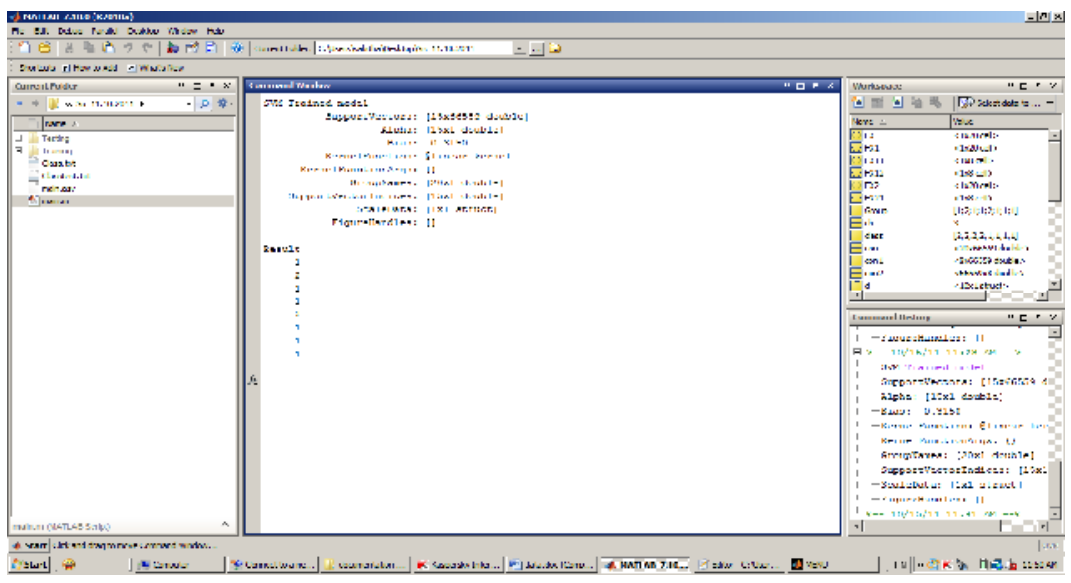

Fig 6: SVM Classifier output

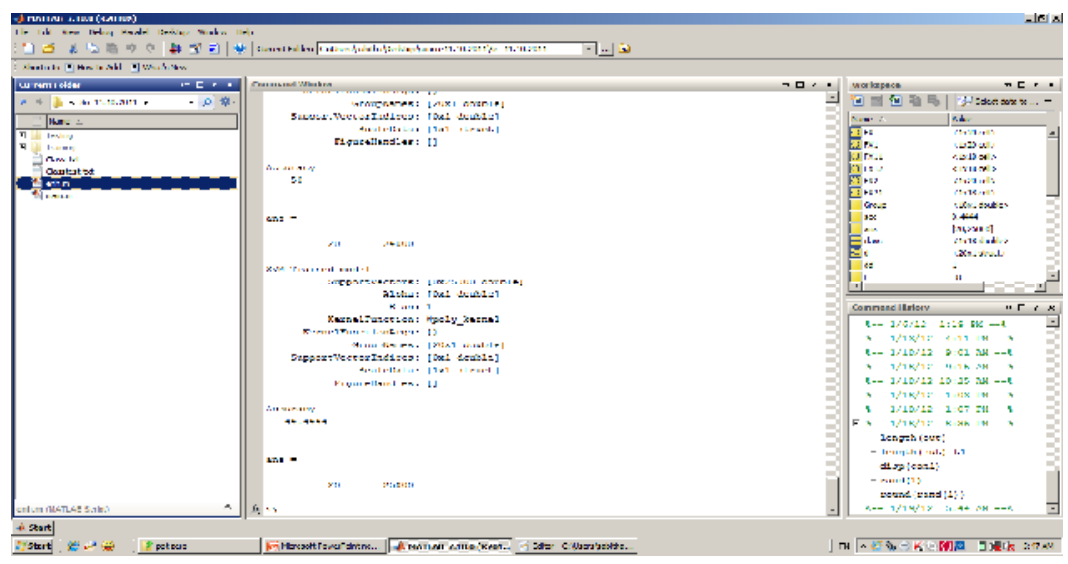

Fig 7 Boosting SVM output 
International Journal of Computer Science \& Information Technology (IJCSIT) Vol 4, No 4, August 2012

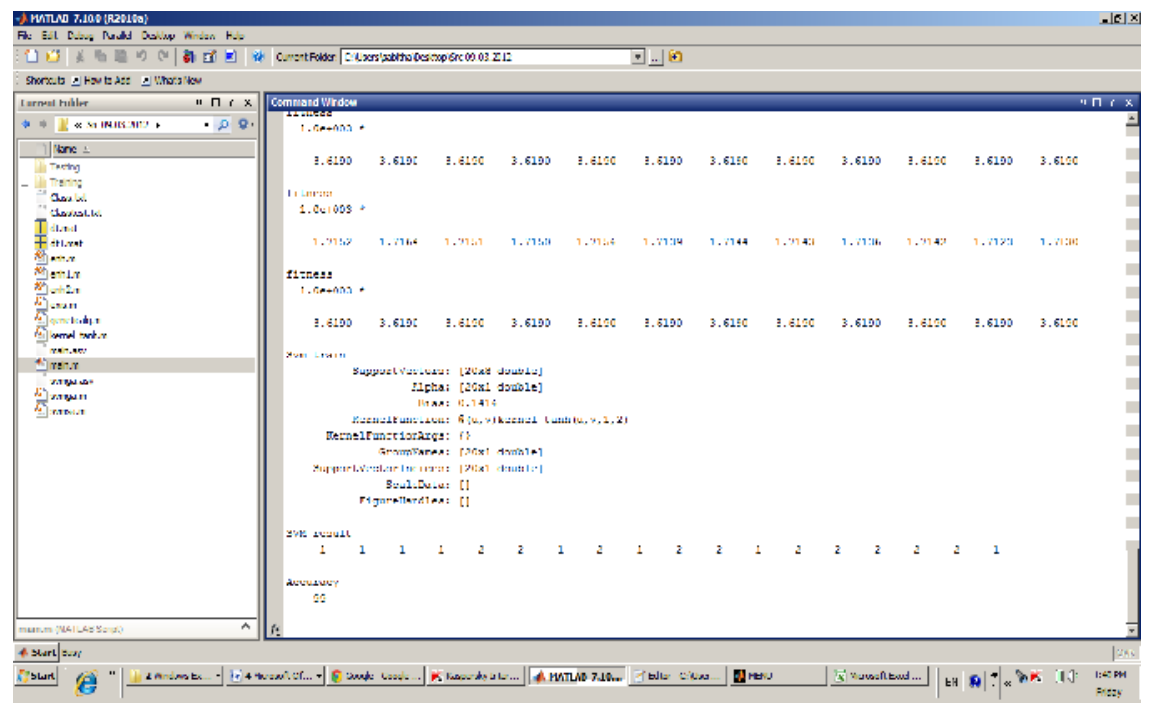

Fig 8 SRM SVM with GA

\section{PERFORMANCE EVALUATION}

The accuracy is calculated for SVM ,Boosting SVM and SRM-SVM with GA classifiers by taking the number of correctly verified individuals to the total number of images taken.

Accuracy $=($ Number of correctly identified images/ Total number of images $) * 100$

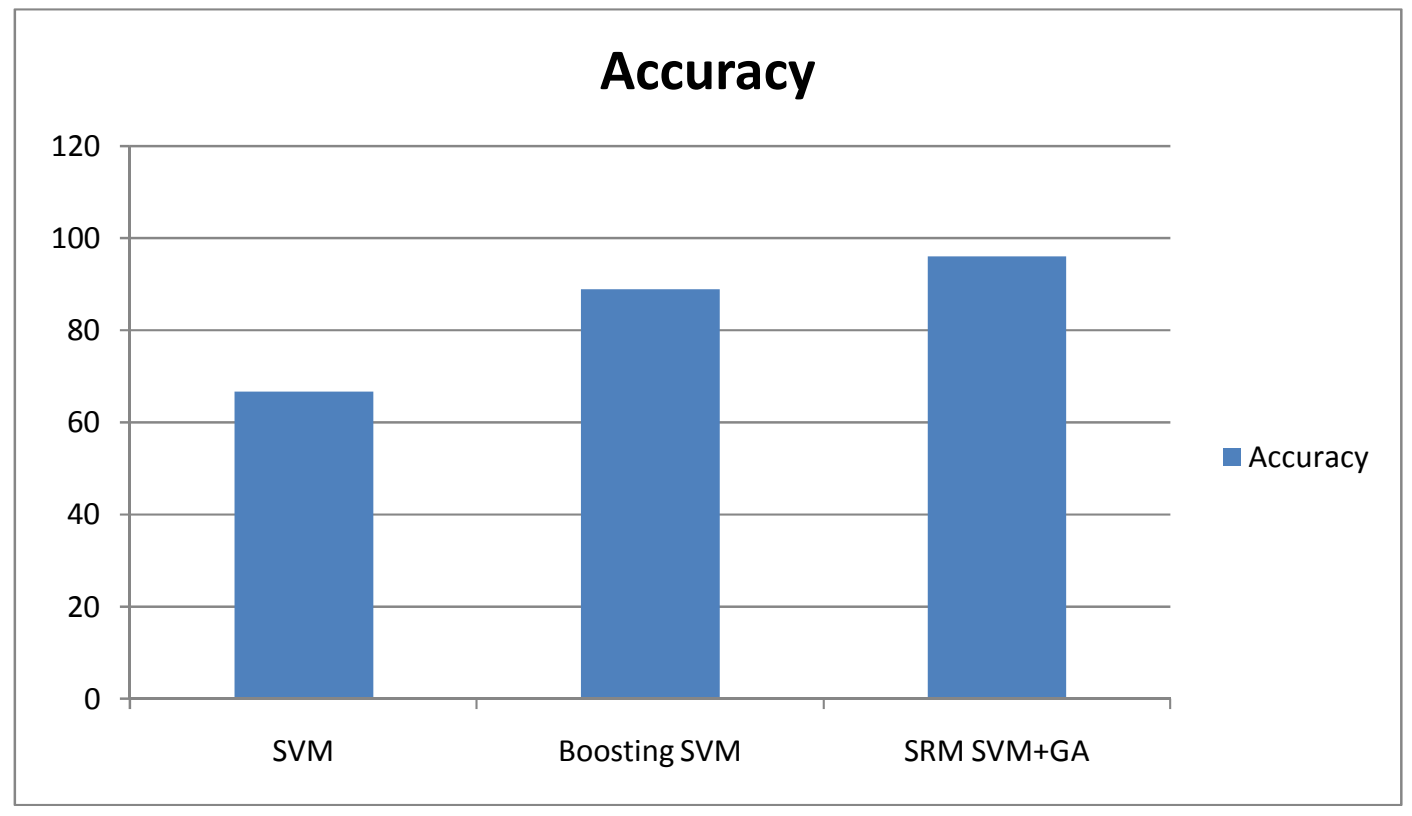

Fig 9 Performance Evaluation 
International Journal of Computer Science \& Information Technology (IJCSIT) Vol 4, No 4, August 2012

\section{Conclusions}

Discriminative methods are used for face verification with age variation. The GOP performs well on face images with large age differences. The boosting techniques and genetic algorithm are used to improve the performance of face verification system. Boosting-SVM increases its capability depends on the number of stages added to the system. SRM-SVM with Genetic algorithm outperformed other techniques such as SVM, Boosting SVM.SVM gives 66.6\% accuracy whereas Boosting SVM gives $88.8 \%$ accuracy. Finally proposed SRM-SVM with GA gives $96 \%$ accuracy.

Support vector machine, Boosting SVM and SRM SVM with GA are used for face verification. Some of the commonly used other classifiers are Bayesian classifier, neural network classifier and decision tree classifier. To further improve the performance other optimization techniques can be apply for classification. Also other representation framework can be apply.

\section{REFERENCES}

[1] Haibin Ling, Member, IEEE, Stefano Soatto, Senior member, IEEE, Narayanan Ramananthan, Student Member, IEEE, and David W. Jacobs ,Member,IEEE(2010)"Face Verification Across Age Progression Using Discriminative Methods".

[2] Hansen F. Chen, Peter N. Belhumeur, David W. Jacobs(2000) "In Search of Illumination Invariants".

[3] Narayanan Ramananthan, Rama Chellappa (2006) "Face verification across age progression" .IEEE Transactions on Image Processing, vol, 15, NO.11.

[4] P. Jonathon Phillips (1999) "Support vector machines applied to face Recognition".

[5] Haibin Ling1,StefanoSoatto2, Narayanan Ramanathan, and David W. Jacobs "A Study of Face Recognition as People Age".

[6] Narayanan Ramanathan Rama Chellappa "Modeling Age Progressionin Young Faces".

[7] Narayanan Ramanathan and Rama Chellappa(2008) "Modeling Shape and Textural Variations in Aging Faces".

[8] Oswaldo Ludwig, Crisstiano Premebida, Urbano Nunes, and Rui Araujo (2011)"Evaluation of Boosting-SVM and SRM-SVM Cascade Classifiers in Laser and vision based Pedestrian Detection".

[9] DARPA. The urban and grand challenges.[online].http://www.darpa.mil/grandchallenge/, (accessed:2010),2003

[10] P. Viola and M. Jones. “ Rapid object detection using a boosted cascade of simple features”. In IEEE Computer Vision and Pattern Recognition(CVPR 01), volume 1, pages 511-518, Hawaii, Dec. 2001.

[11]J. Joines and C. Houck, "On the use of non-stationary penalty functions to solve constrained optimization problems with genetic algorithm," in Proc. 1994 International Symposium evolutionary computation, 1994,pp. 579-584.

[12]L. J. Eshelman and J. D. Schaffer, "Real-coded genetic algorithms and interval-schemata," Foundations of Genetic Algorithms, vol. 2, pp. $187-202,1993$. 


\section{Authors}

Ms.Safiya K.M. was born in Kerala, India, in 1984. She received a Diploma in Information Technology from Govt. Polytechnic, Kerala, India in 2004. She received a B.Tech degree in Information Technology from Mahatma Gandhi University, Kerala, India in 2007. She is currently pursuing M.E in Computer Science and Engineering under Anna University, Coimbatore, India.

Prof..S.Bhuvana received her Bachelor of Engineering degree in Computer Science and Engineering from Sri Ramakrishna Engineering College India, in 1999 and her Master of Engineering degree in Computer Science and Engineering from Kumaraguru college of Technology, India in 2007. At Present, she is working as Assistant Professor in the department of Computer Science \& Engg , Sri Krishna College of Technology, Coimbatore. Currenty she is pursuing her research work in Image Retrieval. Her research interest include Image Processing and machine learning.

Prof. P.Tamije Selvy received B.Tech (CSE), M.Tech (CSE) in 1996 and 1998 respectively. Since 1999, she has been working as faculty in reputed Engineering Colleges. At Present, she is working as Assistant Professor(SG) in the department of Computer Science \& Engg, Sri Krishna College of Technology, Coimbatore. She is currently pursuing Ph.D under Anna University, Chennai. Her Research interests include Image Processing, Data Mining, Pattern Recognition and Artificial Intelligence.

Dr. R. Radhakrishnan is the Principal of Sri Ramakrishna Engineering College. He obtained his Bachelor's degree in Engineering in Electronics and Communication Engineering from Bharathidasan University, received his Masters Degree from P.S.G.College of Technology, Coimbatore, in the year 1997 and the PhD from Anna University Chennai in the year 2008. His research interest includes Wireless Communication, Signal Processing, Networking and Mobile Communication. He has published more than 11 Journal papers in the field of CDMA systems, Mobile communication, Wireless Networking and Signal Processing.
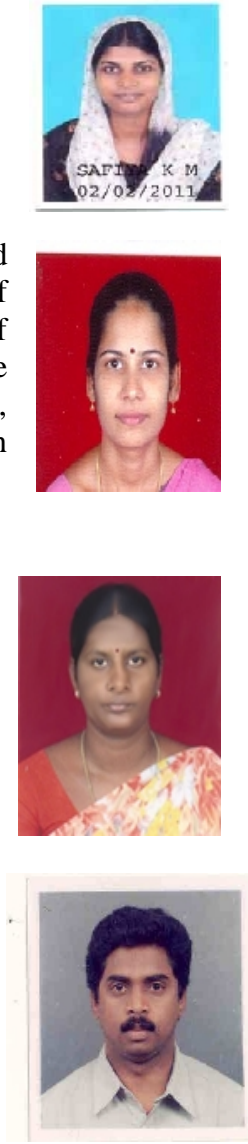